\title{
Nuclear matter calculations with chiral interactions
}

\section{Domenico Logoteta*}

INFN, Sezione di Pisa, Largo Bruno Pontecorvo 3, I-56127 Pisa, Italy

E-mail: domenico.logoteta@pi.infn.it

\section{Ignazio Bombaci}

Dipartimento di Fisica, Universitá di Pisa, Largo Bruno Pontecorvo 3, I-56127 Pisa, Italy INFN, Sezione di Pisa, Largo Bruno Pontecorvo 3, I-56127 Pisa, Italy

E-mail: ignazio.bombaci@unipi.it

\section{Alejandro Kievsky}

INFN, Sezione di Pisa, Largo Bruno Pontecorvo 3, I-56127 Pisa, Italy

E-mail: kievskyedf.unipi.it

\begin{abstract}
We calculate the energy per particle of symmetric nuclear matter and pure neutron matter using the many-body Brueckner-Hartree-Fock approach and employing the Chiral Next-to-next-to-next-to leading order (N3LO) nucleon-nucleon $(\mathrm{NN})$ potential, supplemented with various parametrizations of the Chiral Next-to-next-to leading order (N2LO) three-nucleon force. Such combination is able to reproduce several observables of the physics of light nuclei for suitable choices of the parameters entering in the three-nucleon interaction. We find that some of these parametrizations, provide also reasonable values for the observables of nuclear matter at the saturation point.
\end{abstract}

The 8th International Workshop on Chiral Dynamics, CD2015 ***

29 June 2015 - 03 July 2015

Pisa, Italy

\footnotetext{
*Speaker.
} 


\section{Introduction}

The advent of interactions derived in the framework of chiral perturbation theory (see e.g. [1] and references therein quoted) opened a new and systematic way to investigate low and high energy processes in nuclear physics. The big advantage of using such method lies in the fact that two-body as well as many-body forces can be calculated order by order according to a well defined scheme. This systematic procedure is particularly useful for nucleonic systems where the importance of the three-nucleon force (TNF) is a well established feature. It is indeed well known that high precision nucleon-nucleon $(\mathrm{NN})$ potentials, fitting $\mathrm{NN}$ scattering data up to energy of $350 \mathrm{MeV}$, with a $\chi^{2}$ per datum next to 1 , underestimate the experimental binding energies of ${ }^{3} \mathrm{H},{ }^{3} \mathrm{He}$ by about $1 \mathrm{MeV}$ and that of ${ }^{4} \mathrm{He}$ by about $4 \mathrm{MeV}$ [2]. This missing binding energy can be accounted for by introducing a TNF into the nuclear Hamiltonian [2].

In a previous paper [3], we have investigated whether using the same interactions at two- and three-body level, it was possible to concurrently reproduce properties of finite light nuclei and nuclear matter. Fixing the parameters of the TNF to simultaneously describe the ${ }^{3} \mathrm{H},{ }^{3} \mathrm{He}$ and ${ }^{4} \mathrm{He}$ binding energies and the neutron-deuteron (n-d) doublet scattering length [4], we found that none of the considered interactions was able to reproduce a good saturation point of symmetric nuclear matter. In [3] we used the Argonne18 NN potential [5] supplemented with two different TNFs. In the first case we employed various parametrizations of the Tucson-Melbourne TNF [6], while in the second case, we used the local version of the chiral N2LO [7] TNF (hereafter N2LOL) [8]. In the present work, we consider an interaction fully based on chiral perturbation theory both for the twoand three- nucleon sectors. We use indeed the chiral N3LO [9] NN potential in conjunction with the N2LOL three-nucleon interaction. We note that while the NN potential is calculated at order N3LO of chiral perturbation expansion, the three-nucleon one is calculated at order N2LO. Chiral TNFs have also been calculated at order N3LO [10]. However it seems that their contribution is negligible [11]. Moreover no additional low energy constant appears at this order. Subleading contributions to the TNF come out at order N4LO [12]; their contribution seems to be potentially important. In this work we limit our study considering TNFs calculated at order N2LO.

The paper is organized as follows: in the first section we review the parameters of the N2LOL three-nucleon force and the determination of the low energy constants; in the second section we briefly discuss how to include a TNF in the Brueckner-Hartree-Fock (BHF) approach; finally the third section is devoted to show the results of our calculations and to outline the main conclusions of the present study.

\section{The N2LOL three nucleon force}

Following Ref. [8], for the N2LOL potential we have adopted a cut off of the form $F_{\Lambda}=e^{-\frac{q^{2 n}}{\Lambda^{2 n}}}$ being $q$ the exchanged momentum. We employ $n=2$ and $\Lambda=500 \mathrm{MeV}$. We have then considered five parametrizations of the N2LOL three-nucleon force (hereafter N2LOL1, N2LOL2, N2LOL3, N2LOL4, N2LOL5). For all the five parametrizations, we have kept the values of the parameters $c_{1}, c_{3}$ and $c_{4}$ as the original ones determined in Ref. [9]. These values are reported in the caption of Tab. 1. The values of the low energy constants $c_{E}$ and $c_{D}$ are reported in Tab. 1 . The parametrization $\mathrm{N} 2 \mathrm{LOL} 1$, is the original one proposed in Ref. [8], where $c_{E}$ and $c_{D}$ were fitted to reproduce the 
binding energies of ${ }^{3} \mathrm{H}$ and ${ }^{4} \mathrm{He}$ in a no-core shell model calculation. In Ref. [13], fixing the range of $c_{D}$ between -3 and 3 , the parameter $c_{E}$ was determined fitting the binding energies of ${ }^{3} \mathrm{H}$ and ${ }^{3} \mathrm{He}$. In this way the authors of Ref. [13] obtained two curves $c_{E}\left(c_{D}\right)$ fulfilling the previous constraints. As the two curves turned out to be very close, the authors of Ref. [13] performed an average between them. Finally, for each set of parameters $\left(c_{E}, c_{D}\right)$, the Gamow-Teller (GT) matrix element of Tritium $\beta$-decay was calculated and, using the corresponding experimental value and its error-bars, the minima and a maxima values for $c_{D}$ and $c_{E}$ satisfying this requirement were determined. In the parametrizations N2LOL2 and N2LOL3, we have adopted the minima and the maxima values allowed for $c_{D}$ and $c_{E}$ according to the construction described above. The parametrization N2LOL4, taken from Ref. [14], was obtained in the same way as the N2LOL2 and N2LOL3 ones but allowing that the GT matrix element to be reproduced with a slight larger uncertainty (however less than $1 \%$ ). The last parametrization that we have considered, namely the $\mathrm{N} 2 \mathrm{LOL} 5$, has been obtained fixing the couple $\left(c_{D}, c_{E}\right)$ on the trajectory reproducing the binding energies of ${ }^{3} \mathrm{H}$ and ${ }^{3} \mathrm{He}$ and requiring to get the best saturation density of symmetric nuclear matter.

\section{Inclusion of three-nucleon forces in the Brueckner-Hartree-Fock approach}

As widely discuss in literature $[15,16,17,18]$, three-nucleon forces cannot be included in the BHF formalism $[19,20]$ in their original form. This task would require the solution of a three-body Bethe-Faddeev equation in the nuclear medium and currently this procedure cannot be accomplished. To overcome this problem, an effective density dependent two-body force is built starting from the original three-body one by averaging over the coordinates (spatial, spin and isospin) of one of the nucleons. The effective $\mathrm{NN}$ force due to the general three-nucleon force $W(1,2,3)$ can be written as $[15,21]$ :

$$
W(1,2)=\frac{1}{3} \int d \mathbf{x}_{3} \sum_{c y c} W(1,2,3) n(1,2,3)\left(1-P_{13}-P_{23}\right)
$$

where we have defined $\int d \mathbf{x}_{3}=\operatorname{Tr}_{\left(\boldsymbol{\tau}_{3}, \boldsymbol{\sigma}_{3}\right)} \int d \mathbf{r}_{3}$. In the previous expression $n(1,2,3)$ is the density distribution of the nucleon 3 in relation to the nucleon 1 at $\mathbf{r}_{1}$ and nucleon 2 at $\mathbf{r}_{2}$. The function $n(1,2,3)$ represents the effect of the $\mathrm{NN}$ correlations and will suppress the contributions from the short-range part of $W(1,2,3)$. As in Ref. [3] we assume that this density distribution can be factorized as

$$
n(1,2,3)=\rho g^{2}(1,3) g^{2}(2,3),
$$

where $\rho$ is the nucleon density, $g(1,3)$ and $g(2,3)$ are the correlation functions between the nucleons $(1,3)$ and $(2,3)$ respectively. The latter quantities can be written as $g(1,3)=1-\eta(1,3)$, where $\eta(1,3)$ is the so-called defect function (and similarly for $g(2,3))$. To simplify the numerical calculations and following $[15,3]$, in the present work we use central correlation functions $g(i, j)$ independent on spin and isospin. Moreover, it has been shown $[15,16,17]$ that this central correlation functions, in which are included the main contributions of the ${ }^{1} S_{0}$ and ${ }^{3} S_{1}$ channels, are weakly dependent on the density, and can be approximated by a Heaviside step function $\theta\left(r_{i j}-r_{c}\right)$, with $r_{c}=0.6 \mathrm{fm}$ in all the considered density range. Finally $P_{i j}$ are the spin, isospin and space exchange operators between nucleon $i$ and $j$. 


\begin{tabular}{l|cc}
\hline & $c_{D}$ & $c_{E}$ \\
\hline N2LOL1 & 1.00 & -0.029 \\
N2LOL2 & -0.20 & -0.208 \\
N2LOL3 & -0.04 & -0.184 \\
N2LOL4 & 0.00 & -0.18 \\
N2LOL5 & 0.25 & -0.135 \\
\hline \hline
\end{tabular}

Table 1: Five different parametrizations of the N2LOL three-body force with $\Lambda=500 \mathrm{MeV}$. The values $c_{1}=-0.00081 \mathrm{MeV}^{-1}, c_{3}=-0.0032 \mathrm{MeV}^{-1}, c_{4}=-0.0054 \mathrm{MeV}^{-1}$ have been kept fix in all the five cases. See text for details.

\begin{tabular}{l|cccc}
\hline & $\rho_{0}\left(\mathrm{fm}^{-3}\right)$ & $E / A(\mathrm{MeV})$ & $E_{\text {sym }}(\mathrm{MeV})$ & $L(\mathrm{MeV})$ \\
\hline N2LOL1 & 0.185 & -15.48 & 35.5 & 58.5 \\
N2LOL2 & 0.15 & -11.23 & 29.0 & 44.1 \\
N2LOL3 & 0.15 & -11.96 & 29.3 & 45.0 \\
N2LOL4 & 0.15 & -12.16 & 29.4 & 45.2 \\
N2LOL5 & 0.16 & -13.04 & 31.3 & 48.7 \\
N3LO & 0.41 & -24.25 & 55.0 & 108.2 \\
\hline \hline
\end{tabular}

Table 2: Properties of nuclear matter at saturation point for $\Lambda=500 \mathrm{MeV}$. In the five columns are shown: parametrization of the N2LOL three-body force, saturation density, corresponding value of energy per particle $E / A$, symmetry energy $E_{\text {sym }}$ and slope $L$ of the symmetry energy. Last line refer to the calculation in which just the N3LO NN force has been used. See text for more details.

A different average procedure, with respect to the one discussed above, has been performed in Ref. [22], where an in medium effective NN potential was derived from the N2LO three-nucleon force. In [22] just the on-shell contributions have been determined while the off-shell counterparts have been obtained by extrapolation.

We want to stress that the presence of the exchange operators in the average, neglected in our previous work [3], play a very important role. In this way we obtain a fully density dependent antisymmetric two-nucleon force and we take into account of all internal and external permutation of the TNF. In addition, through this procedure, all operators involved in the TNF contribute to the effective density dependent potential. The resulting effective density dependent potential contains in particular some purely repulsive contributions that are missing when neglecting the $P_{i j}$ exchange operators. Such repulsion is needed to contrast the strong attraction provided at two-body level by the N3LO potential as we will show in next section.

\section{Results and discussions}

We now present the results of our calculations of the energy per particle of symmetric nuclear matter and pure neutron matter using N3LO NN potential supplemented with the N2LOL three-body force. In Fig. 1 we show the energy per particle of pure neutron matter (left panel) and symmetric nuclear matter (right panel) using the cutoff $\Lambda=500 \mathrm{MeV}$. The dotted lines in both plots refer to the calculation performed employing the N3LO potential without any TNF. First we note the huge contribution provided by the TNF to the energy per particle of both symmetric nuclear 

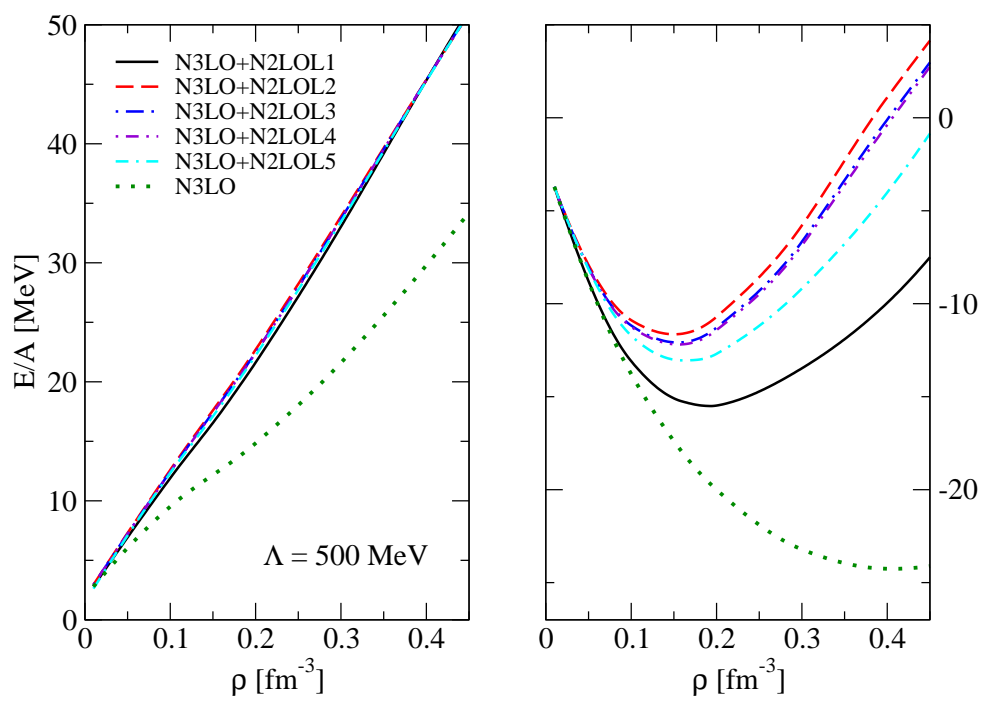

Figure 1: Pure neutron matter (left panel) and symmetric nuclear matter (right panel) versus nucleonic density for the five parametrizations of the N2LOL model. See text for details.

matter and pure neutron matter. In the case of symmetric nuclear matter the saturation point moves indeed from $0.41 \mathrm{fm}^{-3}$ to values between $0.16 \mathrm{fm}^{-3}$ and $0.185 \mathrm{fm}^{-3}$, depending on the specific model used for the TNF (see Tab. 1). More specifically, the parametrization N2LOL1 predicts a reasonable saturation point for symmetric nuclear matter at a density of $0.185 \mathrm{fm}^{-3}$ and an energy per nucleon equal to $-15.48 \mathrm{MeV}$. Referring again to the N2LOL1 model, the shift introduced by the $\mathrm{TNF}$ to the energy per nucleon at the empirical saturation point $\rho_{0}=0.16 \mathrm{fm}^{-3}$ is $\Delta E=2.73 \mathrm{MeV}$ for symmetric nuclear matter and $\Delta E=4.84 \mathrm{MeV}$ for pure neutron matter. On the other hand the parametrizations N2LOL2, N2LOL3 and N2LOL4, all produce a good saturation density of 0.15 $\mathrm{fm}^{-3}$, but the corresponding energy per nucleon is underestimated ranging between $-11.23 \mathrm{MeV}$ and $-12.16 \mathrm{MeV}$ (see Tab. 1). The curves for the energy per nucleon of pure neutron matter are very similar in all the density range considered. We note that the parametrization N2LOL5 is constructed to reproduce the ${ }^{3} \mathrm{H}$ and ${ }^{3} \mathrm{He}$ binding energies and the best saturation density of symmetric nuclear matter. However, we want again to remark that in this case the GT matrix element is not reproduced. It is apparent that none of the present interactions can fulfill the requirement to reproduce simultaneously the ${ }^{3} \mathrm{H},{ }^{3} \mathrm{He}$ and ${ }^{4} \mathrm{He}$ binding energies, the GT matrix element and the saturation point of symmetric nuclear matter. However the parametrization N2LOL1, which has the important property to reproduce ${ }^{3} \mathrm{H}$ and ${ }^{3} \mathrm{He}$ binding energies, provides also a reasonable saturation point for nuclear matter. Such achievements represent a big improvement of our previous calculations [3].

In the case of asymmetric nuclear matter with neutron density $\rho_{n}$, proton density $\rho_{p}$, total nucleon density $\rho=\rho_{n}+\rho_{p}$ and asymmetry parameter $\beta=\left(\rho_{n}-\rho_{p}\right) / \rho$ the energy per nucleon can be accurately reproduced using the so called parabolic approximation [23]

$$
\frac{E}{A}(\rho, \beta)=\frac{E}{A}(\rho, 0)+E_{s y m}(\rho) \beta^{2} .
$$




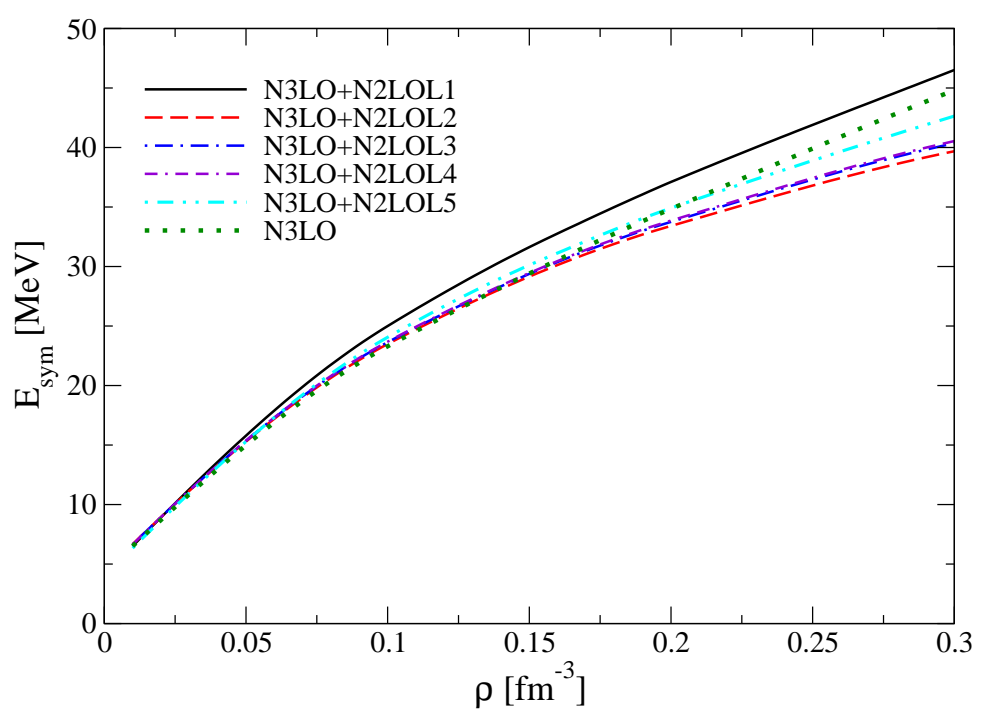

Figure 2: Symmetry energy versus nucleonic density for the five parametrizations of the N2LOL one considered. See text for details.

where $E_{s y m}(\rho)$ is the nuclear symmetry energy. Thus the symmetry energy can be calculated as the difference between the energy per particle of pure neutron matter $(\beta=1)$ and symmetric nuclear matter $(\beta=0)$. The nuclear symmetry calculated with this prescription for various parametrizations of the N2LOL three-nucleon force is plotted in Fig. 2.

To compare our results with the value of the symmetry energy extracted from various nuclear experimental data $[24,25]$, we report in Tab. 2 the symmetry energy and the so called slope parameter

$$
L=\left.3 \rho_{0} \frac{\partial E_{s y m}(\rho)}{\partial \rho}\right|_{\rho_{0}} .
$$

at the calculated saturation density (second column in Tab. 2) for the different TNF models considered in this work. As we can see (Tab. 2) our calculated $E_{\text {sym }}$ and $L$ are in very good agreement with the values extracted from experimental data [24]: $E_{\text {sym }}\left(\rho_{0}\right)=29.0-32.7 \mathrm{MeV}$, and $L=40.5-$ $61.9 \mathrm{MeV}$. This agreement is lost when the TNF is not included in the calculations (see the last row in Tab. 2). Notice that the value of incompressibility $K_{0}$ of symmetric nuclear matter, at the calculated saturation density, is generally quite low. It ranges between $K_{0}=150-170 \mathrm{MeV}$, depending on the TNF model.

Let us now confront our results with those of similar calculations present in literature based on chiral nuclear interactions. The authors of Ref. [14] performed nuclear matter calculations using the BHF approach adopting the effective density dependent NN force derived in Ref. [22]. These authors found results in good agreement with ours both for nuclear matter and pure neutron matter. Several nuclear matter calculations based on chiral interactions have been performed using other many-body techniques. In Ref. [26], using the $V_{\text {lowk }}$-approach and the similarity renormalization group (SRG), it was shown that for a suitable choice of the cutoff $\Lambda_{V l o w k}$ it was possible to repro- 
duce a good saturation point of symmetric nuclear matter keeping for the parameters of the TNF the values fixed in few-body calculations. In Ref. [27], using the self-consistent Green's functions method, it has been found that the saturation point of symmetric nuclear matter is strongly improved with the help of the N2LOL three-nucleon force although, also in this case, the saturation curve results underestimated.

\section{Conclusions}

We have performed several BHF calculations of symmetric nuclear matter and pure neutron matter considering the N3LO NN potential plus the N2LOL three nucleon interaction. The last one has been reduced to an effective density dependent two-body force averaging over the coordinates of one of the nucleons. We want to stress that the parameters of the TNF fitted in calculations of light nuclei have been kept fixed in our nuclear matter calculations. We found that although it was not possible to reproduce the binding energy of ${ }^{3} \mathrm{H},{ }^{3} \mathrm{He}$, the GT matrix element and the saturation point of symmetric nuclear matter simultaneously, one parametrization of the N2LOL three nucleon force, namely the N2LOL1, shows a reasonable saturation point of symmetric nuclear matter as well as values of $E_{\text {sym }}$ and $L$ in good agreement with the experimental ones. These encouraging results spur us towards new combined investigations in few- and many-body nuclear physics using interactions derived in the framework of chiral perturbation theory.

\section{References}

[1] E. Epelbaum, H.-W. Hammer and U. G. Meissner, Rev. Mod. Phys. 81, (2009) 1773.

[2] A. Kievsky, S. Rosati, M. Viviani, L.E. Marcucci and L. Girlanda, J. Phys. G 35, (2008) 063101.

[3] D. Logoteta, I. Vidana, I. Bombaci and A. Kievsky, Phys. Rev. C 91, (2015) 064001.

[4] A. Kievsky, M. Viviani, L. Girlanda and L. E. Marcucci, Phys. Rev. C 81, (2010) 044003.

[5] R. B. Wiringa, V. G. J. Stoks and R. Schiavilla, Phys. Rev. C 51, (1995) 38.

[6] S. A. Coon and H. K. Han, Few-Body Syst. 30, (2001) 131.

[7] E. Epelbaum et al., Phys. Rev. C 66, (2002) 064001.

[8] P. Navratil, Few-Body Syst. 41, (2007) 117.

[9] D. R. Entem and R. Machleidt, Phys. Rev. C 68, (2003) 041001(R).

[10] V. Bernard, E. Epelbaum, H. Krebs and U.-G. Meissner, Phys. Rev. C 77, (2008) 064004; V. Bernard, E. Epelbaum, H. Krebs and U.-G. Meissner, Phys. Rev. C 84, (2011) 054001.

[11] H. Witala, J. Golak, S. Skibinski and K. Topolnicki, J. Phys. 41, (2014) 094011.

[12] L. Girlanda, A. Kievsky and M. Viviani, Phys. Rev. C 84, (2011) 014001.

[13] L. E. Marcucci, A. Kievsky, S. Rosati, R. Schiavilla and M. Viviani, Phys. Rev. Lett. 108, (2012) 052502 .

[14] L. Coraggio, J. W. Holt, N. Itaco, R. Machleidt, L. E. Marcucci and F. Sammarruca, Phys Rev. C 89, (2014) 044321.

[15] B. A. Loiseau, Y. Nogami and C. K. Ross, Nucl. Phys. A401, (1971) 601. 
[16] M. Baldo and L. S. Ferreira, Phys. Rev. C 59, (1999) 682.

[17] X. R. Zhou, G. F. Burgio, U. Lombardo, H.-J. Schulze and W. Zuo, Phys Rev. C 69, (2004) 018801.

[18] W. Zuo, I. Bombaci and U. Lombardo, Eur. Phys. J. A 50, (2014) 12.

[19] M. Baldo, I. Bombaci, G. Giansiracusa, U. Lombardo, C. Mahaux and R. Sartor, Phys. Rev. C 41, (1990) 1748.

[20] M. Baldo, I. Bombaci, L. S. Ferreira, G. Giansiracusa and U. Lombardo, Phys. Rev. C 43, (1991) 2605.

[21] A. Lovato, O. Benhar, S. Fantoni, and K. E. Schmidt, Phys. Rev. C 85, (2012) 024003.

[22] J. W. Holt, N. Kaiser and W. Weise, Phys. Rev. C 81, (2010) 024002.

[23] I. Bombaci and U. Lombardo, Phys. Rev. C 44, (1991) 1892.

[24] J. M. Lattimer, Gen. Rel. Grav. 46, (2014) 1713.

[25] Eur. Phys. J. A 50 (2014) 2, Topical issue on Nuclear Symmetry Energy, edited by B. A. Li, A. Ramos, G. Verde, and I. Vidaña.

[26] K. Hebeler and A. Schwenk, Phys. Rev. C 82, (2010) 014314; K. Hebeler, S. K. Bogner, R. J. Furnstahl, A. Nogga and A. Schwenk, Phys. Rev. C 83, (2011) 031301(R).

[27] A. Carbone, A. Polls and A. Rios Phys. Rev. C 88, (2013) 044302. 